\title{
Analysis on Regional Water Resources Development Threshold Model based on Neural Network
}

\author{
Wang Jianjun \\ School of Civil Engineering, Shandong University; Ji'nan 250100, China.
}

Keywords: Neural Network, Threshold Model, Analysis, Regional Water, Resources Development.

\begin{abstract}
In this literature article, we conduct research on the regional water resources development threshold model based on neural network. With the development of industry and agriculture and the increase of population, water availability will increase, which brings us up out of the water resources characteristics requirements of scientific and reasonable development and utilization of the water resources should be our water conservancy workers focus on research problems in the future, and provide scientific basis for policy makers, and achieve sustainable development. Profound evolvement background based on river basin water resources, as water resources and its exploitation and utilization evaluation has received the full attention in recent years. We combine corresponding principles to propose our perspective that will be innovative.
\end{abstract}

\section{Introduction}

At present, the water resources development and utilization of the environmental impact, from the point of impact the content that can be divided into the ecological variables and the influence of the ecological impact. Including the ecological influences of river water and the sediment conditions, characteristics of water quality and geology, regional climate, the influence of the influence on the ecological variables disappear for the basic biodiversity and ecological function degradation, the two interrelated, interaction, in which the consequences of most affected ecological variables [1-2].

Characteristics and law of water resources has its inherent in its nature refers to the characteristics of applying human activity, no trace, main show is non-uniformity of time and the space distribution, randomness and liquidity, systemic, etc. (1) Terrain and climate differences led to the general uneven space-time distribution of water resource. The non-uniformity of time and space distribution of water resources of the scientific allocation of water resources, improve the efficiency of the water caused great difficulties. (2) The randomness and liquidity. Water randomness is embodied in the evolution of water resources affected by the hydrological random change, year, month between a change in the water all have more or less the same, in a wet year that have the plentiful and mutagenicity, and this change is random. (3) The quality of the gradual change and the renewable. Strictly speaking, the contamination of a certain amount of water that is not known as the water resources that has negative value to human beings. However, the water quality change is a gradual process.

Comprehensive evaluation index system of regional water resources carrying capacity of regional water resources, society, ecology and economy coordinated development situation of comprehensive evaluation and research basis and standards. Determine the comprehensive evaluation index system should make it can comprehensively and objectively reflect China's water resources and coordinated development between society, ecology and the economy development condition, which can guide and supervise Chinese water resource utilization, promote the sustainable development of China, and can make the concept clear and convenient for data collection. As a result, the bearing capacity of water resources research to the national economy development planning, ecological environment protection and sustainable utilization of water resources is of great significance that rise of the water resources carrying capacity research in China in the 80s, after the present rapid vigorous development trend.

In this manuscript, to deal with mentioned challenges, we conduct research on the regional water resources development threshold model based on neural network. In this study, on the core basis of the predecessors' research, comprehensive frequency statistics and theory analysis method to select 
index, namely the current coordinated development evaluation research reports and papers about high frequency statistics to select the indicators, as well as the regional economy, society, ecology and the connotation, characteristics and basic elements of complex system of water resources comprehensive analysis and comparison are made on the main problems, such as, the selection and the harmonious development of closely linked and targeted strong indicators.

\section{Our Proposed Methodology}

\subsection{Neural Network Model.}

Currently in system modeling and the forecasting, applied the most were static multi-layer forward neural network, this is mainly because the network has the ability to approximate any of the nonlinear mapping that by learning. In the following figure, we demonstrate the architecture [3].

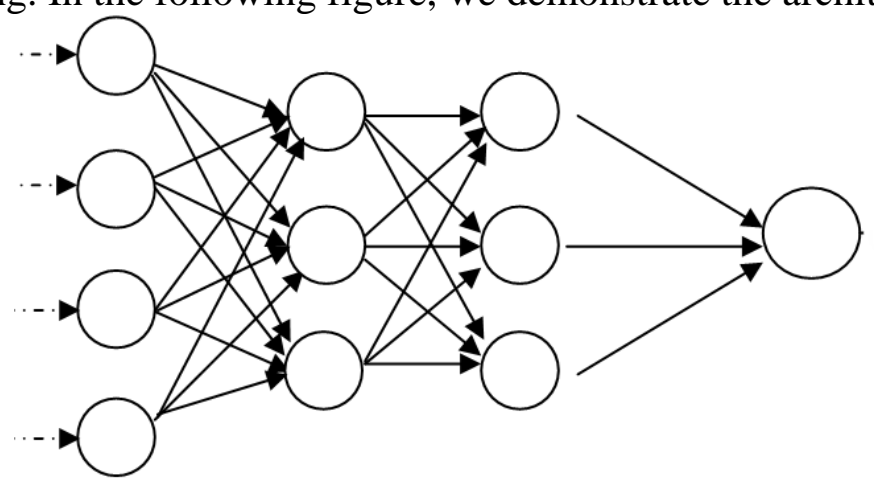

Fig. 1 The General Architecture of the Neural Network Model

BP network is to use Hoff learning algorithm and differentiable nonlinear transfer function of multi-layer network and the typical BP algorithm using the gradient descent method, namely the Hoff algorithm. Now there are many basic optimization algorithm, such as variable metric algorithm and Newton algorithm. The output of the algorithms could be expressed as follows.

$$
f(x)=\sum_{i=1}^{N} w_{i} f_{i}(x)
$$

The improvement of neural network generalization ability and the integration depend on the target space diversity, but the target from the weights of space diversity, space diversity and the regulating object of network training is weight, weight space that has more abundant information than the target space available. In the formula two, we express the object function.

$$
E_{\text {object }}=\frac{1}{2} \sum_{k=1}^{m}\left(y_{p k}-o_{o k}\right)^{2}
$$

Many modal particle swarms in the weight room at the same time, and the track multiple different extreme value point, tracking the same extreme value point belongs to the kind of particles, each type of particle optimal particle as a member of the network. The object function can be revised as below.

$$
E_{\text {object }}^{\prime}=\frac{1}{M} \sum_{k=1}^{N}\left(t_{p k}-o_{o k}\right)^{2}
$$

Neural network is a new branch in the study of neural networks in recent years, is combined with the idea of wavelet transform theory and artificial neural network and construct a new neural network model, it combines good time-frequency localization feature properties of wavelet transform and the self-learning function of network, which has strong approximation ability and fault tolerance.

\subsection{The Complex Network Model.}

Existing in the nature of a large number of complex systems can be described by a variety of basic network as the network is composed of many nodes of connections between nodes and edges. Among them, the nodes represent different individuals in the real system, and represent the link between these individuals. When dealing with multiple edges that one of the most simple way is to combine multiple edge is an edge processing. However, in real life there are some of the edge is not can simple merge processing, such as above mentioned transportation network and communication network, because the network speed of the larger difference between edge to edge. In recent years a large 
number of studies have shown that the topology of the network to the characteristics of the network complex network shows with the classical theory of random graph model of different characteristic [4].

Accordingly, the characteristics could be summarized as follows. (1) Small world network has both with the rule similar features, but also has similar to random networks of the small average path length. (2) Metabolic network, etc., they have power-law degree distribution function form. This in the form of a power-law distribution and fractal self-similar characteristics, no obvious characteristic length, so the class called scale-free network and the formation of the power-law distribution, mainly due to the partial node attached in accordance with the good, the new members of the network nodes tend to be connected with nodes who has a generous. (3) Complex networks due to the non-uniformity degree distribution, lead to face different kinds of basic attack strategy, show different robustness, anti-destroying ability. In the face of core random attack, even though the majority of node failure, breakdown, still can keep the whole network connectivity. (4) Community is a collection of nodes in network, has a close connection between nodes in community, and community for loose connections. It embodies the level of the complex system and module structure.

\subsection{Water Resources Development.}

To solve the problem of the essence of good water, be sure to follow the principle is: balanced and comprehensive treatment, and deal with immediate and long-term, partial and whole, open source and throttling aspects such as relationship, development and protection of water resources argumentation of any development and production project should be that demonstrated in figure two.

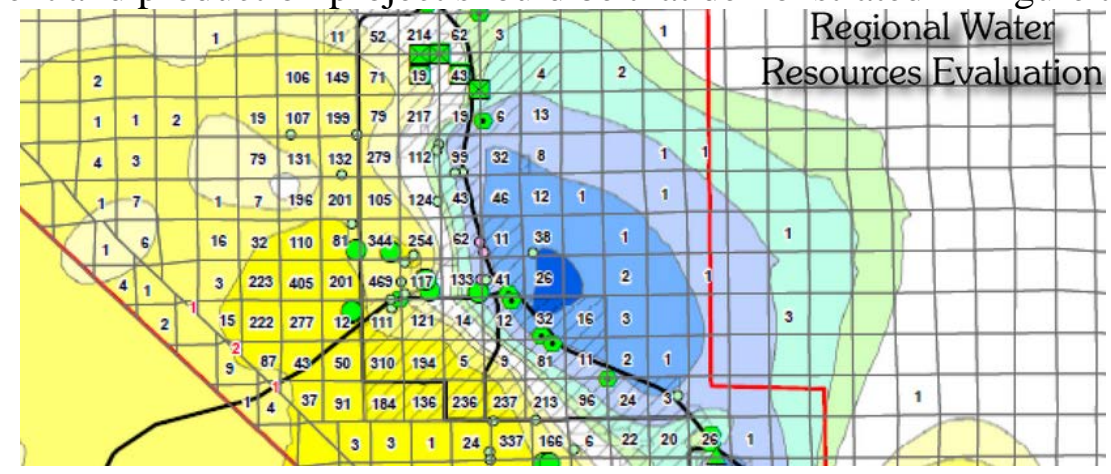

Fig. 2 The Water Resources Development Pattern Demonstration

Profound evolvement background based on the river basin water resources, as the water resources and its exploitation and utilization evaluation has received the full attention in recent years, the index system of research and development is considered to be one of the most effective ways that we should follow the listed guidelines. (1) Water resources system and its development and utilization of the complex, that comprehensive evaluation determines the different aims of water resources and its exploitation and utilization of comprehensive evaluation index and its system is the focus. And due to different regional water resources and its exploitation and utilization of the system and the system environment is different, so the water resources and its exploitation and utilization of comprehensive evaluation index selection is diversity. (2) Water resources and its exploitation and utilization is a complicated system with multiple attribute, involving water conditions, the degree of pattern and the development and utilization of general water resources allocation, the level of economic and social development, ecological environment protection level and the status of the water environment, water resources management, and some other parties. (3) Representative index selection should also be directional and independence, namely to has guiding significance for the direction of the basic water resources and its exploitation and utilization, and should have independence or basic weak correlation between indicators. (4) Water resources evaluation index is an important premise is able to perform numerical calculation, which requires measurability that could reflect the true condition.

\subsection{Threshold Model and Applications.}

In most cases, the water resources carrying capacity is not an area within a numerical as it depends largely on the water resources utilization in water resources management goals. Although the water resources carrying capacity definition involves many factors, focusing on the aspects are different, 
but the meaning of water resources carrying capacity should at least include the following content. (1) The amount of water resources carrying capacity is dynamic. On the one hand, with the progress of project construction and development of technology, the construction of water resources of the local can gradually increase the proportion of total water resources, so as to increase the bearing capacity of the water resources. (2) Water resources carrying capacity is moderate, has the threshold. Considering sustainable development and sustainable utilization of water resources situation, the scale of water resources to support regional economic development and population capacity should have a moderate concept. (3) Natural carrying capacity, it has to do with natural resources, environment and biological processes, such as water resource itself to restore, update, rely on the environment, water environment carrying capacity, etc. It can through expert assessment, complex simulation, with the remote sensing technology and long-term fixed-point observation and other methods to determine [5].
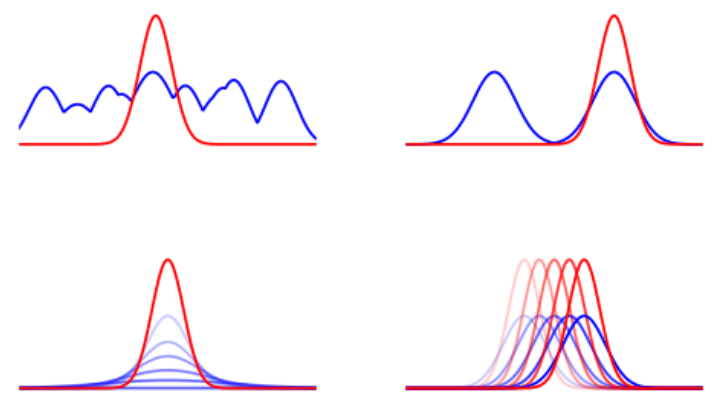

Fig. 3 The Threshold Model Demonstration

\section{Summary}

In this paper, we conduct research and the analysis on the regional water resources development threshold model based on neural network. The national economic and social development of water resources demand and rely on more and more strong, and due to the scarcity of the water resources demand of economic structure and productivity layout must be adapted to water conditions, otherwise leave the security of water resources, all sorts of development are impossible, restricting conditions become a policy and planning of water resources. Therefore, we integrate the neural network to list the enhanced methodology of regional water resources development pattern. In the recent future, we will integrate more corresponding methodologies to form the better analysis on the issues.

\section{References}

[1]. Kuiper, Edward. Water resources development: planning, engineering and economics. Springer, 2013.

[2]. Kling, Harald, Philipp Stanzel, and Martin Preishuber. "Impact modelling of water resources development and climate scenarios on Zambezi River discharge." Journal of Hydrology: Regional Studies 1 (2014): 17-43.

[3]. Cleaver, Frances. Development through bricolage: rethinking institutions for natural resource management. Routledge, 2012.

[4]. Vidic, Radisav D., et al. "Impact of shale gas development on regional water quality." Science 340.6134 (2013): 1235009.

[5]. Wu, Guangyang, et al. "A dynamic model for vulnerability assessment of regional water resources in arid areas: a case study of Bayingolin, China." Water resources management 27.8 (2013): 3085-3101. 